\title{
The effects of concentration based on the absorbance form the Ultraviolet- Visible (UV-VIS) spectroscopy analysis
}

\author{
Jamal Moammar Aldabib ${ }^{* * 10}$, Mohamed Faraj Edbeib ${ }^{2}$ \\ ${ }^{1}$ Department of Dental Technology, Faculty of Medical Technology, Bani Walid University, \\ Bani Walid/Libya \\ ${ }^{2}$ Department of Animal Production, Faculty of Agriculture, Bani Walid University, Bani \\ Walid/Libya
}

\section{Abstract}

This experiment utilizes the material characterization technique known as UV-Vis spectroscopy. The aim of this research is to study the UVVis absorption spectroscopy readout in order to investigate the effect of concentration on absorbance for gold solution and to determine the value of band gap for powder sample $\left(\mathrm{TiO}_{2}\right)$. The types of $\mathrm{TiO}_{2}$ specimens can be characterized on a solid form of powder, thin film and/or in a liquid form. The preparation of each state of specimen are varies. In this current research, Liquid samples of $\mathrm{Au}$ nanoparticles dispersed in different concentrations of ethanol were characterized using UV-Vis technique. The absorbance versus concentration plot of the Au-ethanol samples were found to obey Beer's law. When examining the solid state in a form of $\mathrm{TiO}_{2}$ powder, the sample need to be packed before running the experiment. To calculate the band gap of each matter from the reflectance data of UV-Vis, the graph of energy $(\mathrm{eV})$ versus is $\left[\ln \left(\frac{R_{\max }-R_{\min }}{R-R_{\min }}\right)\right]^{2}$ plotted. The J-curve is identified quickly, extrapolating the tangent to intersect the $\mathrm{x}$-axis to obtain the band gap value.

\section{Introduction}

Ultraviolet visible (UV-Vis) spectroscopy have been widely used to provide characteristic information for about any type of materials. For example, UV-Vis spectroscopy can be used to observe organic or inorganic, solid or liquid groups such as organic molecule and functional group (Aimanant and Ziemann, 2013; Ranney and Ziemann, 2016), reflectance measurements for coatings, paints, textiles (Bisulca et al., 2008), biochemical analysis (Schmid, 2001), dissolution kinetics (Jargalan et al., 2015), band gap measurements (Dharma

\footnotetext{
${ }^{1}$ Correspondence: jamalald71@gmail.com
} 
et al., 2009) etc. These informations are provided by the UV-Vis depending on the extent of absorbance or transmittance of a different wavelengths beam light and the different response of samples (Thomas and Causse, 2017).

\subsection{Electromagnetic Spectrum}

Spectroscopic processes rely on the fact that electromagnetic radiation interacts with atoms and molecules in discrete ways to produce characteristic absorption or emission profiles. The property of electromagnetic radiation (EMR) that determines the range of colour perceived is wavelength. The part of the electromagnetic spectrum that the eye can detect is known as the visible region. EMR may be simply represented as a sine wave. These visible wavelengths cover a range from approximately 400 to $800 \mathrm{~nm}$ (Thomas and Causse, 2017).

Basically, when measuring the optical density using spectrophotometers, visible light corresponds to a particular wavelength or color, is absorbed and disappears. The remaining light, lacking this color, shows the remaining mixture of colors as non-white light. Figure 1 illustrates the approximate complementary relationship between the wavelengths of light absorbed and the wavelengths transmitted. For example, in a blue substance, there would be a strong absorbance of the complementary (opposite it in the color wheel) color of light, orange (Sommer, 2012).

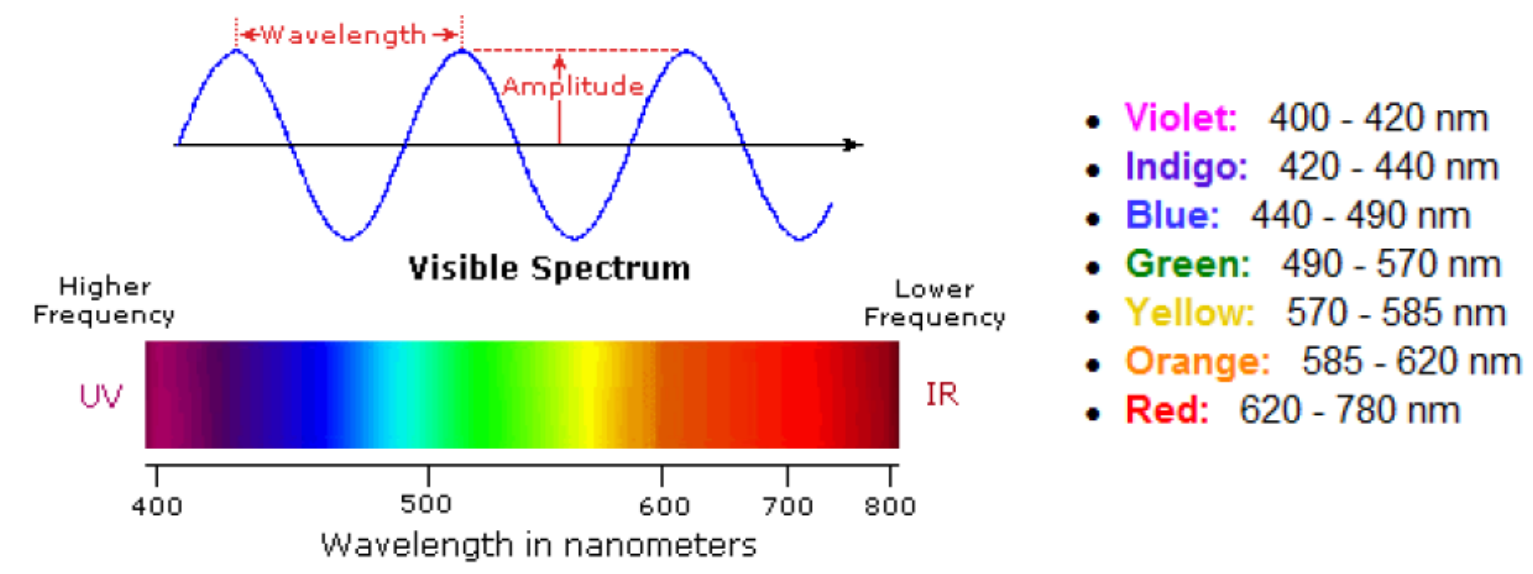

Figure 1. Sine wave representation of electromagnetic radiation and electromagnetic spectrum 
Wavelength is the distance between adjacent peaks or troughs (Figure 2). The wavelength, $\lambda$, of EMR can be expressed as a function of its frequency, $v$, and the speed of light, $c$, by the following simple equation:

$$
v=\frac{c}{\lambda}
$$

EMR behaves as a particle and as a wave (the dual nature of light), and the wavelength of such a particle, a photon, is related to energy by the equation:

$$
E=\frac{h c}{\lambda} 10^{9}
$$

Where $\mathrm{h}$ is the Planck's constant $(6.63 \mathrm{x} 10-34 \mathrm{Js}), \mathrm{c}$ is the speed of light in vacuum $(2.998$ $\mathrm{x} 108 \mathrm{~ms}-1), \mathrm{E}$ is the energy of the photon and is the wavelength in $\mathrm{nm}$.

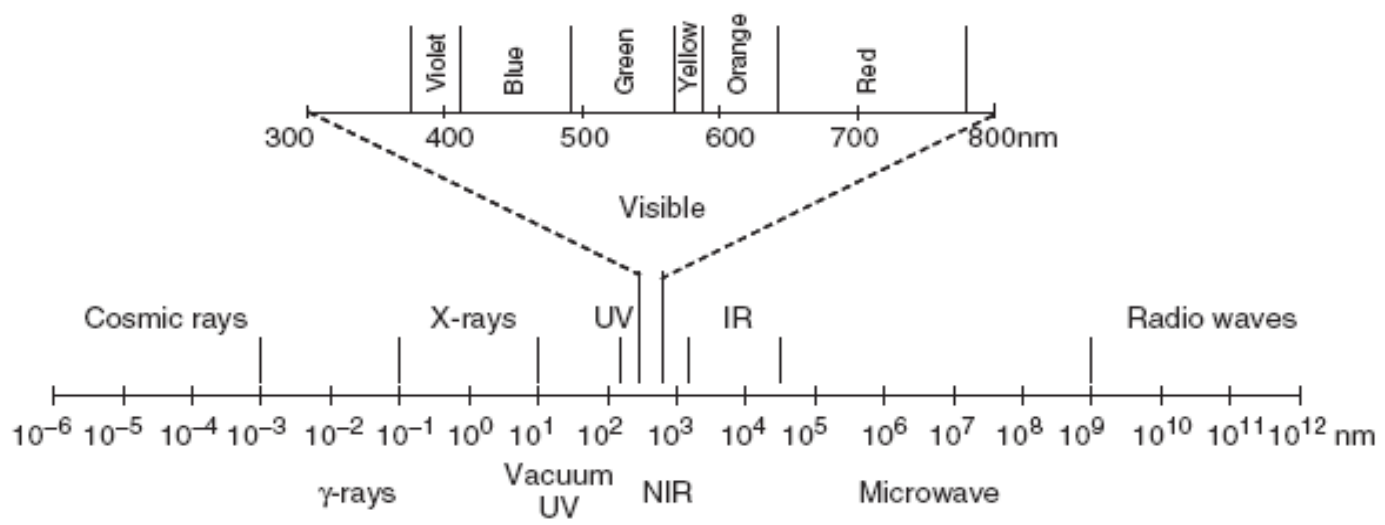

Figure 2. The electromagnetic spectrum (Thomas and Causse, 2017)

\subsection{Electronic Transition}

When an atom or molecule absorbs energy, electrons are promoted from their ground state to an excited state. In a molecule, the atoms can rotate and vibrate with respect to each other. These vibrations and rotations also have discrete energy levels, which can be considered as being packed on top of each electronic level. The absorption of UV or visible radiation corresponds to the excitation of outer electrons. In this analysis on the transitions involving $\pi$, $\sigma$ and $n$ electrons will be considered (Kalantar-zadeh, 2008). 
Table 1. Electronic energy levels and transtitions (Kalantar-zadeh, 2008)

\begin{tabular}{ccc}
\hline Transition & Region of electronic spectra & Example \\
\hline$\sigma \rightarrow \sigma^{*}$ & Vacuum ultraviolet & $\mathrm{CH}_{4}$ at $125 \mathrm{~nm}$ \\
$\pi \rightarrow \pi^{*}$ & Far-ultraviolet, sometimes near-ultraviolet & Acetone at $190 \mathrm{~nm} ;$ \\
& methylamine at $213 \mathrm{~nm}$ \\
$\mathrm{n} \rightarrow \sigma^{*}$ & Ultraviolet & Saturated aldehydes at $180 \mathrm{~nm}$ \\
$\mathrm{n} \rightarrow \pi^{*}$ & Near-ultraviolet and visible & Acetone at $277 \mathrm{~nm} ;$ \\
& & nitroso-t-butane at $665 \mathrm{~nm}$
\end{tabular}

\subsection{Beer's Law}

When a beam of electromagnetic radiation is passed through an absorbing substance, the intensity of the incident radiation (Io) will be greater than that of the emergent radiation (I). The absorption of radiant energy by matter can be described quantitatively through the general principle known as Beer's law. Beer's law states that the amount of radiation absorbed (absorbance, A) or transmitted by a solution or medium is directly proportional to the concentration of the absorbing substance present, $\mathrm{c}$ (moles per liter) and the path length of radiation through the sample, $\mathrm{b}$ (centimeters). Hence, plot of absorbance versus concentration should give a straight line passing through the origin with slope equal to $\mathrm{Cb}$ (Gary and O’Reilly, 1986).

$\mathrm{A}=-\log \left(\frac{I}{I o}\right)=\mathrm{Cbc}$, where $\mathrm{C}=\mathrm{k} / 2.303$

The constant $\epsilon$ is called the molar absorptivity and is independent of the concentration and path length (Kalantar-zadeh, 2008). Beer's law equation can be used to determine the concentration of an organic substance by identifying its maximum absorbance in the UV-Vis absorption spectrum, provided that the path length and molar absorptivity are known (Kalantar-zadeh, 2008).

\subsection{Mechanism of Double Beam UV-VIS Spectroscopy}

The function of this instrument is relatively straightforward. A beam of light from a visible and/or UV light source (red) is separated into its component wavelengths by a prism or diffraction grating. Each monochromatic (single wavelength) beam in turn is split into two equal intensity beams by a half-mirrored device. One beam, the sample beam (magenta), 
passes through a small transparent container (cuvette) containing a solution of the compound being studied in a transparent solvent. The other beam, the reference (blue), passes through an identical cuvette containing only the solvent. The intensities of these light beams are then measured by electronic detectors and compared. The intensity of the reference beam, which should have suffered little or no light absorption, is defined as Io. The intensity of the sample beam is defined as I. Over a short period of time, the spectrometer automatically scans all the component wavelengths in the manner described. The ultraviolet (UV) region scanned is normally from 200 to $400 \mathrm{~nm}$, and the visible portion is from 400 to $800 \mathrm{~nm}$ (Pavia et al., 2008).

Different methods have been widely used to determine concertation of a substance in solution. Such as, acid-base titration to find the concentration of the hydrogen ion (determining the $\mathrm{pH}$ of solution). There are other properties of a solution can be changed with concentration e.g. density, conductivity and colure. Beer's law relates colour intensity and concentration. Comparing to $\mathrm{pH}$ titration, The use of colour-based method can be much faster especially when there are samples with different concentrations of the same substances. (Fujishima et al., 2000; Nakata and Fujishima, 2012).

$\mathrm{Au}$ nanoparticles and $\mathrm{TiO}_{2}$ have always been one of the most important topics in the research of photocatalysts due to its special activity and stability (Bickley et al., 1991). According to Zeng et al. (2019) and Padikkaparambil, Narayanan, Yaakob, Viswanathan, and Tasirin (2013) it has always been difficult to obtain nano- $\mathrm{TiO}_{2}$ with a small particle size, high dispersion and high photocatalytic activity. In this study, the spectroscopy (UV-vis), was used to obtain pure $\mathrm{TiO}_{2}$ with a small particle size, good dispersibility. Owing to its relatively high intrinsic band gap, Ultraviolet (UV) irradiation is required for $\mathrm{TiO}_{2}$ photoactivity.

\section{Materials and Methods}

\subsection{Sample preparation}

$\mathrm{UV}-\mathrm{V}$ is can be used to characterize organic and inorganic samples in the form of gaseous, liquid or solid. Each different type of materials mentioned needs different sample preparation techniques. In this experiment, two types of samples were analyzed. The first type is Gold (Au) nanoparticles obtained from Sigma Aldrich (St. Louis, USA). Au was suspended in 
ethanol solution (Sigma-Aldrich, USA) with four different concentrations ( $2 \mathrm{ppm}, 5 \mathrm{ppm}, 10$ ppm, and $20 \mathrm{ppm}$ ) respectively, which may be differentiated by the intensity of purple color. After the sample is properly poured into cuvette, it is ready to be sent for characterizing using UV-Vis instrument Lambda 35 UV-vis spectrophotometer (Perkin-Elmer Inst., Norwalk, CT). The effect of concentration on absorbance was investigated. Absorption edge is expected to be observed at particular wavelength. For this sample, the liquid used in the reference sample has to be the same with that of which the Au nanoparticles diluted with. Examples of solution available are water, alcohol, acetone, chloroform, and toluene. In this experiment, the solution used is ethanol thus the reference sample is ethanol. The second type sample is the powder sample of $\mathrm{TiO}_{2}$ (Sigma-Aldrich, USA). For the powder sample, the sample needed to be compacted into the sample holder before it can be analyzed and no sample reference is needed.

\subsection{Instrumental Principle}

In this experiment, is used to carry out the samples characterization. The light from the source, after passing through the monochromator (prism), is split into two separate beams; one for the sample and the other for the reference (Kalantar-zadeh, 2008). The absorption signal produced by the reference cell is automatically subtracted from the absorption signal produced by the sample cell, giving a net signal that corresponds to the absorption of the sample solution (Pavia et al., 2008). As a result, a plot of UV-Vis spectra (absorbance or reflectance versus wavelength) will be generated in the monitor of computer.

\subsection{Data Interpretation}

From the raw data for the Au nanoparticles, the absorbance peak of each concentration is plotted against the respective concentration of the solution. The data for $\mathrm{TiO}_{2}$ is initially generated in the form of wavelength against reflectance. The data for wavelength is converted into energy using the equation and the reflectance is calculated by $\left[\ln \left(\frac{R_{\max }-R_{\min }}{R-R_{\min }}\right)\right]^{2}$. 


\section{Results and Discussion}

\subsection{The Effect of Sample Concentration on the Absorbance}

$\mathrm{UV}-\mathrm{Vis}$ analysis is able to provide valuable information on the size, structure and aggregation properties of gold nanoparticles. The absorbance is an important parameter that can be used to calculate the nanoparticle concentration or estimate the nanoparticle size.

Figure 3 shows the results for wavelength against absorption (absorption curves) for $\mathrm{Au}$ nanoparticles suspended in ethanol solution with concentrations 2 ppm, 5 ppm, 10 ppm, and $20 \mathrm{ppm}$; from the wavelength of $438.8 \mathrm{~nm}$ to $600 \mathrm{~nm}$. The peak wavelengths of each curve are labeled in the Figure 3.

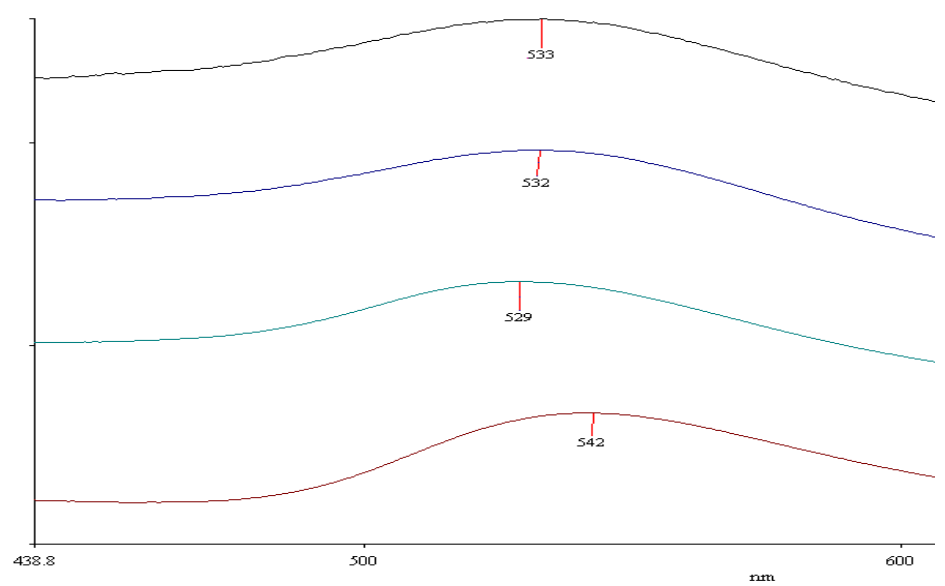

Figure 3. UV absorption spectra of Au nanoparticles dispersed in ethanol with different concentration from wavelength of $438.8 \mathrm{~nm}$ to $710.2 \mathrm{~nm}$

According to Beer's law, the absorbance should be propositional to the concentration. However, the gold solution with 20 ppm shows the highest absorptance value (Figure 4). This concentration values is also the highest. The lowest value of the gold solution with $2 \mathrm{ppm}$ is also has the lowest concentration. This means that the effect of the concertation on the absorbance obey the Beer's law. 


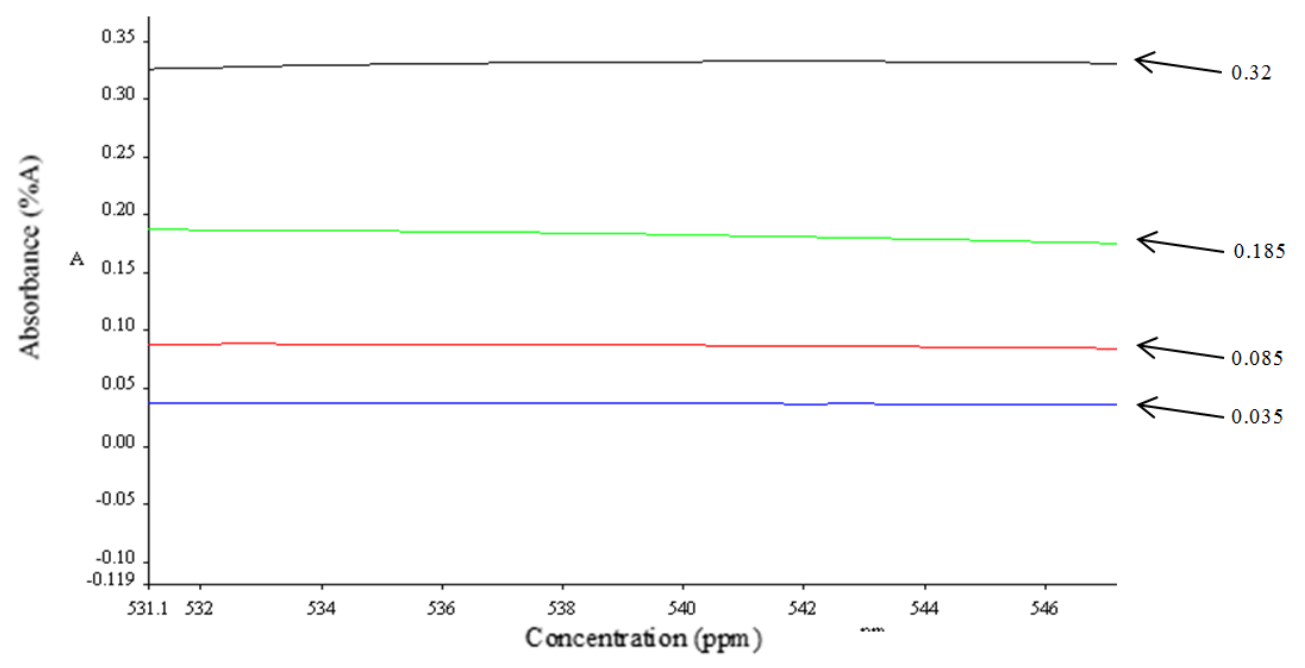

Figure 4. Graph of absorbance versus concentration from $531.1 \mathrm{~nm}$ to $546 \mathrm{~nm}$

Figure 5 shows the plot of concentration versus absorbance for the Au ethanol solution. The plot is a straight line, thus satisfying Beer's law. This plot can be used to predict the concentration of an unknown Au ethanol solution, with the absorbance results obtained from the UV-Vis analysis (Liu et al., 2007).

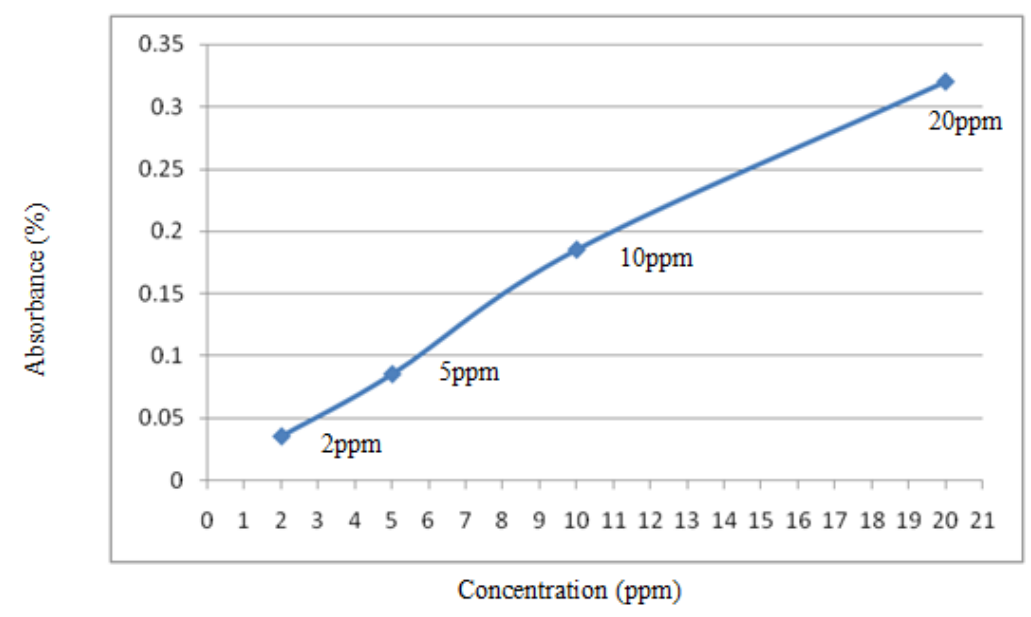

Figure 5. Graph of concentration versus absorbance obeying Beer's law

\subsection{Band gap measurement of powder sample: $\mathrm{TiO}_{2}$}

$\mathrm{UV}-\mathrm{V}$ is is an important material characterization technique to determine band gap of a material. $\mathrm{TiO}_{2}$ is semiconducting material used in optoelectronic devices (Singla et al., 2009). The band gap of the semiconducting material $\mathrm{TiO}_{2}$ is determined from the data obtained from 
UV-Vis analysis. The data obtained is tabulated in terms of energy $(\mathrm{eV})$ and $[\ln (\mathrm{Rmax}-$ $\mathrm{R} \min ) /(\mathrm{R}-\mathrm{R} \min )]^{2}$.

Figure 6 shows the plot of energy $(\mathrm{eV})$ against $[\ln (\mathrm{Rmax}-\mathrm{Rmin}) /(\mathrm{R}-\mathrm{Rmin})]^{2}$ for $\mathrm{TiO}_{2}$ in the full $\mathrm{eV}$ range and as well as the $\mathrm{J}$-curve region. The $\mathrm{J}$-curve is used to determine the band gap values by extrapolating the tangent of the J-curve.

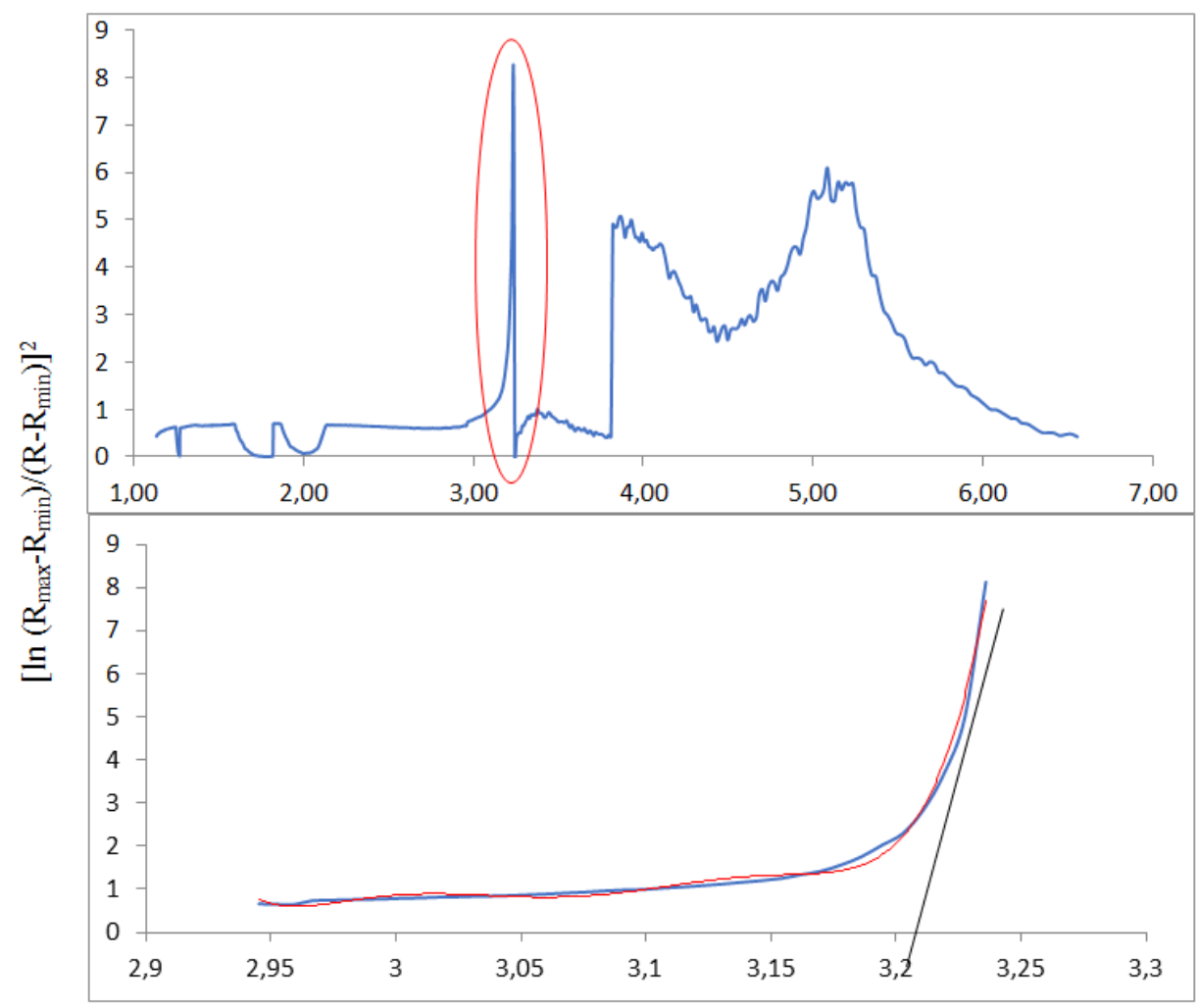

Energy (eV)

Figure 6. (a) Graph of $[\ln (\mathrm{Rmax}-\mathrm{Rmin}) /(\mathrm{R}-\mathrm{Rmin})]^{2}$ versus energy $(\mathrm{eV})$ for $\mathrm{TiO}_{2}$; (b) $\mathrm{J}$-curve identified from the circled area

From the $\mathrm{J}$-curve of $\mathrm{TiO}_{2}$ the band gap value of the respective sample is determined (Table 2). The theoretical value for the band gap is compared to the one which was determined from this experiment. 
Table 2. Experimental and theoretical band gap values of $\mathrm{TiO}_{2}$ (Singla et al., 2009)

Sample $\quad$ Experimental band gap value $(\mathrm{eV}) \quad$ Theoretical band gap value $(\mathrm{eV})$

$\begin{array}{lll}\mathrm{TiO}_{2} & 3.20 & 3.18\end{array}$

There is a slight difference between the theoretically and experimentally determined band gap values. This difference is most probably due to the misalignment of mirrors as monochromator in the instrument or errors during the sample preparation process.

As such, precautionary measure is to be taken before starting the experiment. The alignment of instrument has to be checked before characterization being carried out. Powder sample must be compacted sufficiently in order to give precise analysis and results.

\section{Conclusion}

$\mathrm{UV}-\mathrm{V}$ is spectroscopy is a technique which utilizes the light absorption principle to characterize materials. This technique is useful in predicting the effects of concentration based on the absorbance form the UV-Vis analysis. As such, it is a very useful for evaluating the direct band gap of materials. Different sample types would require different preparation techniques. In this experiment, liquid samples and powder sample were used. In this experiment, gold solutions with 4 different concentrations are analyzed using UV-Vis technique. In accordance to Beer's law, the concentrations are directly proportional to the absorbance. The band gap values for $\mathrm{TiO}_{2}$ powder is determined experimentally to be $3.20 \mathrm{eV}$.

\section{References}

Aimanant, S., Ziemann, P.J. 2013. Development of spectrophotometric methods for the analysis of functional groups in oxidized organic aerosol, Aerosol Science and Technology, 47(6): 581-591.

Bickley, R.I., Gonzalez-Carreno, T., Lees, J.S., Palmisano, L., Tilley, R.J. 1991. A structural investigation of titanium dioxide photocatalysts, Journal of Solid State Chemistry, 92(1): 178-190.

Bisulca, C., Picollo, M., Bacci, M., Kunzelman, D. UV-Vis-NIR reflectance spectroscopy of red lakes in paintings. 9th International Conference on NDT of Art, 25-30 May 2008, Jerusalem, Israel. 
Dharma, J., Pisal, A., Shelton, C. 2009. Simple method of measuring the band gap energy value of $\mathrm{TiO}_{2}$ in the powder form using a UV/Vis/NIR spectrometer. Application Note Shelton, CT: PerkinElmer.

Fujishima, A., Rao, T.N., Tryk, D.A. 2000. Titanium dioxide photocatalysis, Journal of photochemistry and photobiology C: Photochemistry Reviews, 1(1): 1-21.

Gary, D., O’Reilly, E. 1986. Instrumental analysis: Boston, London, Sydney, Toronto: Allyn and Bacon Inc.

Jargalan, N., Tropin, T., Avdeev, M., Aksenov, V. 2015. Investigation of the dissolution kinetics of fullerene C 60 in solvents with different polarities by UV-Vis spectroscopy, Journal of Surface Investigation. X-ray, Synchrotron and Neutron Techniques, 9(1): 12-16.

Kalantar-zadeh, K., and Fry, B. 2008. Nano fabrication and patterning techniques. In: Kalantar-zadeh, K., Fry, B. (Eds). Nanotechnology-Enabled Sensors. Boston, MA: Springer US. pp. 135-210.

Liu, X., Atwater, M., Wang, J., Huo, Q. 2007. Extinction coefficient of gold nanoparticles with different sizes and different capping ligands, Colloids and Surfaces B: Biointerfaces, 58(1): 3-7.

Nakata, K., Fujishima, A. 2012. $\mathrm{TiO}_{2}$ photocatalysis: Design and applications, Journal of photochemistry and photobiology C: Photochemistry Reviews, 13(3): 169-189.

Padikkaparambil, S., Narayanan, B., Yaakob, Z., Viswanathan, S., Tasirin, S.M. 2013. $\mathrm{Au} / \mathrm{TiO}_{2}$ reusable photocatalysts for dye degradation, International Journal of Photoenergy, 2013: 1-10.

Pavia, D.L., Lampman, G.M., Kriz, G.S., Vyvyan, J.A. 2008. Introduction to spectroscopy: Cengage Learning. United States of America.

Ranney, A.P., Ziemann, P.J. 2016. Microscale spectrophotometric methods for quantification of functional groups in oxidized organic aerosol, Aerosol Science and Technology, 50(9): 881-892.

Schmid, F. X. 2001. Biological macromolecules: UV-visible spectrophotometry. eLS.

Singla, M.L., Shafeeq, M.M., Kumar, M. 2009. Optical characterization of ZnO nanoparticles capped with various surfactants, Journal of Luminescence, 129(5): 434-438.

Sommer, L. 2012. Analytical absorption spectrophotometry in the visible and ultraviolet: the principles (Vol. 8). Elsevier.

Thomas, O., Causse, J. 2017. Chapter 2 - From spectra to qualitative and quantitative results. In: Thomas, O., Burgess, C. (Eds). UV-visible spectrophotometry of water and wastewater (Second Edition). Elsevier. pp. 37-72.

Zeng, G., Zhang, Q., Liu, Y., Zhang, S., Guo, J. 2019. Preparation of $\mathrm{TiO}_{2}$ and $\mathrm{Fe}-\mathrm{TiO}_{2}$ with an impinging stream-rotating packed bed by the precipitation method for the photodegradation of gaseous toluene, Nanomaterials, 9(8): 1173. 